\title{
Die Diastase der Bauchmuskeln im Wochenbett.
}

Von

\section{Prochownick}

in Hamburg.

Von Alters her haben die Geburtshelfer dem Auseinanderweichen der Bauchmuskeln in der Schwangerschaft und den auf dieses bezogenen Folgeerscheinungen, z. B. der Schiefheit und dem sogenannten Bruch der Gebärmutter ihre Aufmerksamkeit zugewendet. Diese Aufmerksamkeit ist mehr eine diagnostische, als eine auf Heilung bestrebte gewesen und es ist dies ja bei der Schwierigkeit, solchen Leiden während zunehmender Schwangerschaft abzuhelfen, leicht erklärlich.

Um so mehr muss es auffallen, dass in der Pathologie und Therapie des Wochenbettes bei uns zu Lande, in der Lehre und in der Praxis auf die Heilung der Bauchmuskeldiastase ein verhältnissmässig geringes Gewicht gelegt wird. Der fortgesetzte Kampf für die Einführung der antiseptischen Maassregeln und der Umstand, dass die Folgeerscheinungen der Diastase sich vorerst dem Auge des Geburtshelfers, sowohl in Anstalten, als in der praktischen Thätigkeit entziehen, machen diesen Mangel begreiflich. Allein je mehr wir uns bestreben in die Geburtshülfe prophylaktische Therapie einzuführen, $d$. h. bei der Behandlung von Geburt und Wochenbett bewusst einer Reihe von Erkrankungen vorzubeugen, welche später ätiologisch auf das Wochenbett bezogen werden, um so mehr sollten auch die am äusseren Leibe vorkommenden krankhaften Zustände klinisch beachtet und einer vorbeugenden Behandlung unterzogen werden.

Wir haben dazu besondere Veranlassung, weil die socialen Zustände unseres Vaterlandes den Frauen des Volkes viel weniger Ruhe und Rücksicht auf die eigene Person gestatten, als mehrfach anderwärts. Aber auch in die mittleren und selbst in die höheren Stände hinauf erstreckt sich bei uns die Beobachtung, 
dass es eine ganz ausserordentlich grosse Zahl von Frauen giebt, welche ,gleich von der ersten Entbindung her einen starken Leib behalten haben's. Bei den letzteren kann dies gewiss nicht, wie man von den Frauen der arbeitenden Klassen anzunehmen berechtigt wäre, auf Mangel an Ruhe, zu frühes Aufstehen, Rockbänder, Schnürleibdruck und dergleichen bezogen werden, sondern muss auf einer nicht genügenden oder unrichtigen Beachtung des Leibes im Anschlusse an den Gebäract beruhen. Ich habe iiber diesen Punkt eine Reihe von Erhebungen auf einer Reise durch die grösseren Städte Englands angestellt, und es ergab sich, dass in den niederen Volksschichten in ungleich geringerer Zahl als bei uns Frauen mit aufgetriebenem Leibe zu finden sind, und dass dieses Phänomen in den höheren Ständen, innerhalb welcher durchschnittlich mehr Kinder geboren werden, als bei uns, geradezu auffallend selten ist. ${ }^{1}$ ) Ich bin iberzeugt, dass nicht das in England allgemein übliche längere Liegen und die relativ bessere Wöchnerinnenpflege auch der Aermeren ${ }^{2}$ ) davon allein die Ursache sind, sondern dass in dem festgewurzelten Gebrauche, baldigst nach der Entbindung Tücher oder Binden möglichst passend um den Leib herum zu legen und selbige auch nach dem Aufstehen noch eine Zeit lang zu tragen, die bessere Annäherung der Bauchmuskeln und die seltenere Dickleibigkeit der englischen Frauen beruht. Dem etwaigen Einwande, dass hierbei noch die Beckenform und die Grössen- resp. Gewichtsverhältnisse der Kinder zu berücksichtigen sind, sei gleich dạmit begegnet, dass die Beckenform und Beckenneigung der Engländerinnen, an lebendem und skelettirtem Material gelegentlich anderer Arbeiten von mir untersucht, wenigstens zu Norddeutschland keine hervorragenden Unterschiede aufweist und dass die englischen Kinder in Grösse und Gewicht, auch in den Anstaltsbeobachtungen aus den niederen

1) Die Beobachtungen wurden gesammelt theils in den reichgefültten poliklinischen Wartesälen der grossen Hospitäler, theils auf eigens zu diesem Zwecke unternommenen Wanderungen durch die besonders volkreichen und ärmeren Quartiere der englischen Grossstädte. In beiden Fällen haben mich englische Collegen in liebenswürdiger Weise unterstützt.

2) In den mit Entbindungsanstalten versehenen Grossstädten, z. B. London, sind die Mehrzahl aller Insassen stets Frauen der niederen Stände, in denjenigen, welche - (zum Theil principiell, wie Birmingham) - solche Institute nicht besitzen, wird den Frauen von den grossen Hospitälern aus auch für normale Entbindungen ausreichend ärztliche Hülfe zu Theil. In beiden Fällen wird für möglichst langes Liegen und gute Pflege Sorge getragen und stets dem Vermeiden der Bauchdiastase Beachtung zugewendet. 
Prochownick, Die Diastase der Bauchmuskeln im Wochenbett. 421

Ständen, den unseren eher voran- als nachstehen. Es bedarf wohl kaum der Erwähnung, dass die jenseits des Kanales üblichen Maassregeln natürlich nicht immer genügen und dass auch dort die Folgen starken Auseinanderweichens der Bauchmuskeln sich in einer Reihe von Fällen geltend machen.

Allein es braucht nicht nur das Schönheitsgefühl uns zu vorbeugenden Bestrebungen gegen die „Dickleibigkeit“ Anlass zu sein. Die Erfahrungen nach Bauchschnitten haben uns im Laufe der letzten Jahrzehnte mit den vielen Beschwerden und Nachtheilen der sogenannten Bauchbrüche genügend bekannt gemacht. Hegar hat gewiss mit Recht darauf hingewiesen und in die rechten Worte gefasst, was Viele mit ihm fühlten, dass „einige, manchmal zuerst in ihren Entstehungsursachen dunkele gynäkologische Erkrankungen auf die Erschlaffung der Bauchwand und mit ihr des Bauchfelles zurückgeführt werden können". $\mathrm{Er}$ schliesst seinen bezüglichen Vortrag ${ }^{1}$ ) mit der Anschauung, dass „diese Anomalie ohne Zweifel sehr vernachlässigt" sei und dass viel Schaden verhütet würde, ,wenn man im Wochenbett und kurz nachher gegen die Relaxation der Bauchdecken und des Beckenbodens die gehörigen Maassregeln ergriffe!“

Endlich aber treten schon im Wochenbett selbst mitunter Krankheitserscheinungen auf, welche die nicht völlige Ungefährlichkeit der Banchmuskeldiastase beweisen und einer erhöhten Aufmerksamkeit auf dieselbe das Wort reden. Zwei Beobachtungen mögen dies erhärten.

I. Frau Kr., Kaufmannsgattin, entband ich im Februar 1884 vom ersten Kinde. Erste Kopflage, erste Schädelstellung. Kleine (ca. $152 \mathrm{~cm}$ ), kräftige, gesunde Frau von 25 Jahren. Normales Becken. Etwas verfrühter Abfluss des Fruchtwassers, verzögerte Geburt durch langsame Mutterhalserweiterung, Gesammtdauer 22 Stunden. Einmal 0,015 Morphium subcutan wegen krampfhafter Wehen. Sehr energisches Mitarbeiten der Frau in den letzten zwei Stunden. Leichte Beckenausgangszange wegen Gefahr für das Kind durch mehrfache Nabelschnurumschlingung. Kein Chloroform; trotz Abmahnung besonders heftige Bauchpresse während der Operation. Grosses, starkes Kind, $3700 \mathrm{~g}$, Knabe. Kurz vor der Zangenanlegung und zwei Mal darnach je $2 / 3 \mathrm{~g}$ Mutterkorn in Form von Fluidextract. Ein-

1) Ueber einige Folgezustände hochgradiger Erschlaffung der Beckenbauchwand. Deutsche med. Wochenschrift 1884, S. 572 (Nr. 36). 
seitige Incision nach rechts mit zwei Jodoformseidesuturen vereinigt. Einmalige vaginale Sublimatirrigation $(1: 4000)$.

Siebzehn Stunden nach der Entbindung bei normaler Temperatur und ruhigem, vollen Pulse, der nur bei den Schmerzanfällen von 84 auf 96 ansteigt, um dann bald wieder abzufallen, stellen sich böchst intensive Leibschmerzen mit schnell zunehmender Auftreibung, Uebelkeit, Würgen, zwei Mal geringes Erbrechen ein. Die Anfälle treten kolikartig mit versehieden langen Pausen auf, die stärkste Empfindung ist um den Nabel berum und vom Nabel abwärts. Bei ganz gesund befundenem Genitalapparate wird 0,4 Calomel verordnet. Nach fünf Stunden hat sich der Zustand eher versehlimmert, als verbessert; die Schmerzanfälle sind heftig, Temperatur normal, Puls 96. Ein genaues Durchfühlen des stark aufgetriebenen, nur auf der Höhe und um den Nabel herum sehr empfindlichen Leibes ergab jetzt, dass zwischen die stark wulstigen, über $12 \mathrm{~cm}$ auseinanderstehenden Ränder der angespannten, sich derb und hart anfühlenden Musculi recti eine beträchtliche Menge aufgeblähter Darmschlingen hindurchgedrängt war. Es zeigte sich zugleich, dass seitlich im Leibe fast gar kein Meteorismus und geringe Schmerzhaftigkeit vorlag. Nach sofortiger Anwendung von zwölf Tropfen Opiumtinctur per anum und bald darauf weiteren zehn Tropfen per os liessen die Symptome in einer halben Stunde nach, ebenso verlor sich die heftige Contractur der Muskelbäuche und es gelang, die Därme zurückzubringen; eine anderweitige peritoneale Bruchpforte als die weisse Linie war nicht nachzuweisen. Der Zwischenraum zwischen den Recti wurde mit Gaze leicht ausgefuillt und ein Heftpflasterverband darüber gelegt. Unter mehrfacher Wiederholung des Verbandes erfolgte Heilung.

Bemerkt sei noch, dass bei der jungen Frau das Missverhältniss zwischen der kurz und gedrungen gebauten Mutter und dem langen, kräftigen Kinde sich in besonders starker Anspannung der Banchhaut kundgab; stark entwickelte Striae in grosser Ausbreitung erstreckten sich bis zur unteren Grenze des oberen Schenkeldrittels.

Genau dasselbe Verbalten bot die zweite Wöchnerin dar, deren Krankengeschichte sich in vielen Punkten an die erste anlehnt.

II. Frau Hr., Kaufmannsgattin, die Tochter eines geburtshülflich vielbeschäftigten Collegen, entband ich in Gemeinschaft mit dem Vater im September 1884. Knapp mittelgrosse, kräftige Frau von 22 Jahren. Geburt etwas verzögert, langsame Erweiterung des unteren Segmentes bei normalem Becken und gut vorbereiteten Weichtheilen. In den letzten Stunden äusserst lebhafte Action der Bauchpresse. Spontangeburt in erster Schädellage. Gleich nach der Geburt im Verlauf der nächsten Stunden nach und nach fünf Theelöffel von einer Mischung aus 2,0 Extr. Secal. cornut. (Pharmacop. german.) und 50,0 Aq. Cinnamomi. Nachdem der erste Tag des Wochenbettes sehr gut verlaufen war, traten gegen Ende desselben heftige Leibschmerzen mit schnell zunehmendem Meteorismus in typisch-kolikartigen, zuerst Nachwehen vortäuschenden Anfällen auf mit mehrfachem Erbrechen und dauernder Uebelkeit. Temperatur und Puls 
gut, Genitalbefund normal; wegen Harnverhaltung zwei bis drei Mal in 24. Stunden Katheterismus nothwendig. ${ }^{1}$ )

0,3 Calomel und kalte Umschläge verschlimmern den Zustand, so dass der Vater noch in der Nacht zu Opiumsuppositorien à 0,04 und Priessnitz'schen Einwickelungen ïberging und damit Besserung erzielte. Am anderen Morgen ergab bei erneutem Schmerzanfall eine sorgfältige Palpation genau denselben Befund, wie im ersten Falle, und eine Zurückdrängung der zwischen den hart und fest contrahirten Banchmuskeln hervorquellenden geblähten Darmschlingen führte unter mehrmaliger Anwendung von Opium and Wärme zum Verschwinden der Schmerzen. Dieselben kehrten, da die Wöchnerin gegen Leibbinden sehr empfindlich war, noch zwei Mal beim resp. nach dem Stuhldrängen (unter Beihülfe von Abführmitteln) in geringerem Grade wieder, jedes Mal von Durchtritt einiger Darmschlingen zwischen die straff gespannten Bauchmuskeln begleitet. Erst in der dritten Woche wurde ein Heftpflasterverband gut vertragen, und gelang es allmälig, die noch immer weite Spalte zwischen den Musculi recti zu verringern.

Fassen wir die charakteristischen Momente dieser beiden Fälle kurz zusammen, so beobachteten wir:

Junge, kräftige, muskulöse primipare Frauen; kräftige grosse Kinder, etwas reichlich gross im Verhältniss zum kleinen Bauchraum der Mutter. Höchst entwickelte Striae; verzögerte Geburt mit langsamer Erweiterung des Gebärmutterhalses, Neigung zu Krampfwehen und hochgradige Anspannung der Bauchpresse. Gegen Ende des ersten Tages nach der Entbindung plötzlich eintretende Schmerzhaftigkeit des Leibes in kolikartigen Anfällen, besonders dicht unterhalb des Nabels, mit schnell wachsender Darmauftreibung, Uebelkeit, Würgen, selbst Erbrechen, bei normaler Temperatur und nahezu regulärem Pulse; Verschlimmerung des Gesammtzustandes bei Anwendung eines Abführmittels. Starke Anspannung der weit auseinanderklaffenden, aber heftig contrahirten und im ganzen Verlaufe durch ihre harten wulstigen Ränder leicht palpabeln Musculi recti abdominis. Hochgradige Hervorwölbung aufgetriebener Darmschlingen mit sichtbarer Peristaltik zwischen den Muskelbäuchen nebst besonderer Empfindlichkeit im Bereiche der Tympanie bei gleichzeitiger Weichheit, Eindrückbarkeit und weit geringerer Schmerzhaftigkeit der Bauchseitenwände. Nachlass sämmtlicher Erscheinungen auf Opium und

1) Die Wöchnerin hatte schon vor der Verheirathung und während der Schwangerschaft mehrfach an rechtsseitiger Nierenkolik und mässigem, aber langdauerndem Blasenkatarrh gelitten. 


\section{Prochownick, Die Diastase der Bauchmuskeln im Wochenbett.}

Wärme; zugleich nach diesen Mitteln leichte Reposition der vorgelagerten Intestina.

Wir haben somit ein ganz klares, gut charakterisirtes Krankheitsbild vor uns, welches diagnostisch nicht besonderen Schwierigkeiten unterliegt. Zuerst kann der Symptomencomplex, namentlich wenn bei ängstlichen oder nervösen Personen der Puls mehr durch die peritoneale Reizung beeinflusst würde, als es in unseren Fällen geschah, recht erschreckend wirken; eine genaue Untersuchung wird aber schnell die gewünschte Aufklärung bringen.

Es scheinen nun Fälle mit so ausgeprägten Erscheinungen, wie die geschilderten, selten vorzukommen, wenigstens konnte ich in der mir zugängigen Literatur keine beschrieben finden; in geringerem Grade treten sie gewiss öfter auf und werden unter der Annahme von Nachwehen, Blähungen, Stuhlverhaltung abgethan; wenigstens ist mir dies, seitdem ich mit Aufmerksamkeit darauf geachtet habe, mehrfach rorgekommen. Sicherlich erweisen diese Vorfälle, dass auch acute Krankheitszustände durch die Diastase der Bauchmuskeln im Wochenbette auftreten können, und führen uns damit auf die Nothwendigkeit, dieselbe prophylaktisch zu beachten, hin.

Gehen wir nun zurörderst den Ursachen der Bauchmuskeldiastase nach, so ist vorauszuschicken, dass wir mit der Bezeichnung Diastase und besonders mit der gemeiniglich gebrauchten ,Diastase der Recti" lediglich ein Symptom, nicht eine Krankheit charakterisiren.

Die anatomische Ursache $^{1}$ ) des Auseinanderweichens beruht nicht auf den Musculi recti selbst, sondern auf der mit wachsendem Bauchinhalt und Bauchdruck zunehmenden Dehnung und Anspannung der gesammten, ganz vorzugsweise aber der transversalen Muskulatur, also der beiden äusseren und inneren Obliqui. Den natürlichen Bedingungen an der (aufrecht gehenden) Frau entsprechend concentrirt sich dieser Druck zuerst auf die vielfache Sehnendurchkreuzung der queren Bauchmuskulatur, die Linea alba, welche Hyrtl so zutreffend mit dem Rohrstuhlgeflecht vergleicht. Durch Vermittlung dieses Zuges und Druckes auf die weisse Linie tritt dann eine Uebertragung auf die mit derselben eng zusammenhängende fibröse Scheide (Vagina recto-abdominalis) der longi-

1) Man vergleiche hierüber, sowie in Betreff der Literatur die betr. $\mathrm{Ka}$ pitel in den anatomischen Lehr- und Handbüchern, besonders bei Luschka und Henle. 
tudinalen Muskeln ein, indem an derselben mit zunehmender Spannung der Aponeurosen ein centripetaler Zug erfolgt. Dieser ruft das mechanische Auseinandertreten der Muskelbäuche der Recti hervor.

Ausser auf die Mittellinie muss aber der stetig wachsende Bauchdruck allmälig auch auf die Muskulatur selbst wirken. Znnächst wird die Elasticität derselben durch beständige starke Anspannung herabgesetzt; bei starkem Missverhältniss zwischen Bauchraum und Bauchinhalt oder schnell wiederholten starken Ausdehnungen folgen dann druckatrophische Veränderungen der Muskeln. In beiden Fällen werden vorwiegend die Obliqui in ihrer Gesammtmasse betroffen. Die Längsmuskeln sind bei ihrer geringen Breite, "ihrer Faserrichtung; ihrer relativen Dicke und ihrer eigenartigen Durchwirkung mit den sehnigen Inscriptionen dem a tergo wirkenden Drucke weniger ausgesetzt und sie weichen daher meistens, lediglich dem Zuge ihrer fibrösen Scheiden folgend, auseinander, ohne in sich stärkeren Aenderungen ihres Tonus oder ihrer Gewebsmasse so ausgesetzt zu sein, als die transversale Muskulatur. ${ }^{1}$ ) Nur bei hochgradigsten Bauchausdehnungen (Zwillinge, Hydramnios u. s. w.) unterliegen auch die Recti der Druckatrophie.

Die pathologische Anatomie liefert uns über die Richtigkeit dieser Anschauung bisher wenig Aufschluss, allein wir können uns in der klinischen Beobachtung Ersatz verschaffen; am klarsten aus der vergleichsweise herangezogenen operativen Gynäkologie. Machen wir den Bauchschnitt bei grossen (namentlich gleichmässig flüssigkeitserfüllten) Geschwülsten, so ist von einer Bauchmuskulatur, auch von Recti kaum noch die Rede; mit der weissen Linie wird der letzte, höchst gespannte Halt durchtrennt, sofort wölbt sich der Bauchinhalt durch die auch bei kleinerem Schnitte weitklaffende, schlaffrandige Wunde hindurch. Handelt es sich um mittelgrosse Tumoren, besonders feste, so sind fast stets die Recti gut erhalten, meist ein wenig auseinandergezogen, so dass doch die Linea alba noch sofort sichtlich ist, nur die seitliche Bauchwand erweist sich als schlaff, der Schnitt muss, um eine genügende

1) Allerdings spricht Hyrtl anmerkungsweise auch von einem Breiterwerden und bedeutender Veriängernng der Recti in der Schwangerschaft; allein es existiren besonders über das Breiterwerden bis jetzt keine exacten Bestimmungen und es muss fraglich sein, ob es sich nicht um einfache Abplattung bei dem Zug seitens der fibrösen Scheide handelt. 


\section{Prochownick, Die Diastase der Bauchmuskeln im Wochenbett.}

Breite der Wunde zu erlangen, meist grösser sein, als man vorher nach der Grösse des zu entfernenden Objectes taxirt hat, fast stets ist eine manuelle Nachhülfe zur Auseinanderspannung der angelegten Oeffnung nöthig. Hat man endlich eine Castration, Salpingotomie, Nephrektomie zu vollziehen, so vermehren sich die Schwierigkeiten, indem gewöhnlich auch die seitliche Muskulatur straff und elastisch ist: die Linea alba muss selbst von Geübten mitunter gesucht werden ${ }^{1}$, der Schnitt relativ gross sein, besonders wenn Beckentoilette nöthig wird; die Hand des Operirenden wird beim Einführen und Manipuliren oft direct unangenehm durch die Muskelspannung belästigt; der sonst gern wegen des Haltes, welchen er der Bauchaponeurose von unten her verleiht, gemiedene Musculus pyramidalis muss meist gespalten werden, in Einzelfällen ist selbst Tenotomie der Recti unumgänglich.

Auch auf geburtshülflichem Gebiete finden wir klinische Beweispunkte für unsere Meinung. Bei Mehrgeschwängerten, besonders solchen mit schnell aufeinanderfolgenden Schwangerschaften, genügt schon ein Vergleich der Bauchmuskulatur in liegender und sitzender Stellung, um diese Verhältnisse zu klarer Anschauung zu bringen, indem sich vorzüglich in den mittleren Schwangerschaftsmonaten (sechsten bis achten) die gut erhaltenen, scharf angespannten Muskelbäuche der Recti in toto charakteristisch von der sonst schlaffen Bauchwandung herausheben. In vielen Fällen wölben sich in derselben Weise und ebenso scharf sichtbar und tastbar die ileocostalen Partien des Quadratus lumborum hervor, den Luschka als Rectus abdominis posticus an Werth und Bedeutung dem Rectus der Vorderbauchwand gleichgestellt wissen will. Zwischen den so angespannten Längsmuskeln kann man dann die Dürftigkeit und Schlaffheit der queren Muskellagen durch die Untersuchung erkennen.

Hat uns nun die bisherige Betrachtung gelehrt, dass der Krankheitsbegriff ,Diastase der Bauchmuskeln" lediglich ein symptomatischer ist, so brauchen wir doch diese eingebürgerte Bezeichnung nicht aufzugeben, denn das Symptom der Diastase ist ein so hervorragendes, dass es den wichtigsten Maassstab für unsere Erkenntniss des Zustandes und die Anpassung unserer Heilbestrebungen abgiebt. Wir besitzen nämlich keine genauen Untersuchungen über die mechanisch-physikalischen und histolo-

1) Falls man überhanpt Gewicht darauf legt, den Schnitt genau durch die weisse Linie zu führen. 
gischen Veränderungen der queren Bauchmuskeln im Verlaufe der Schwangerschaft; wir besitzen nur einige wenige Messungen über die Ausdehnungsgrade der Linea alba und die Entfernungen der inneren Rectusränder (Cruveilhier). Allein auch wenn wir solche Messungen besässen, würden sie uns bei der grossen Zahl der individuell verschiedenen Möglichkeiten wenig klinisch-diagnostischen Nutzen bieten, und so bleibt für eine grosse, vielleicht die grösste Mehrzahl der Fälle die fühl- und messbare Diastase der Recti bei der einzelnen Person unser wichtigster Maassstab, um die Stärke der Sehnenanspannung zu erkennen und aus dieser Rückschlüsse auf die Muskelbeschaffenheit zu machen. Natürlich gilt dies nur für die Schwangerschaft, und jetzt erst nach Erledigung dieser mehr anatomischen Prämissen legen wir uns die Frage vor: Wie gestalten sich die Verhältnisse nach der Entbindung der Frau? Dieselben werden sich auf Grund unserer Erörterungen gestalten je nach dem Verhältniss zwischen Bauchraum und Bauchinhalt, und als modificirender Factor wird dazu unter Umständen die muskuläre Arbeitsleistung bei der Geburt selbst hinzutreten.

In der Norm ist das physiologische Missverhältniss zwischen Raum und Inhalt gering, weder dieses noch die Geburt erschöpft die Elasticität und den Tonus der Bauchmuskulatur, die oft gar nicht vorhandene, mitunter einige Centimeter betragende Rectaldiastase klappt gewissermaassen zusammen, fast sofort oder im Laufe weniger Stunden post partum, und der Leib bietet in kurzer Frist ein ganz normales Palpationsergebniss. Bei so günstigen Verhältnissen sieht man hier und da Frauen, deren Abdomen selbst nach zwei und drei Wochenbetten (wenn sie nicht zu schnell einander folgten) ein so intactes Aussehen und Gefühl darbietet, dass selbst ein Geübter in Zweifel gerathen kann, ob die Betreffende geboren habe.

Von dieser Norm zweigen sich nun zahlreiche Varietäten ab. Es kann nicht im Sinne dieser Zeilen liegen, dieselben je nach der Combination der oben berührten ursächlichen Momente theoretisch zu erörtern, sondern es möchte das richtigere sein, die wichtigsten klinischen Bilder, zu denen dieselben führen, festzuhalten und an diese dann die Therapie anzupassen. Ich glaube, wir können drei Zustände klinisch einigermaassen genau trennen, und trotzdem in einzelnen Fällen ihre Grenzen und Uebergänge zweifellos sich verwischen und schwierig zu erkennen sind, als 
428 Prochownick, Die Diastase der Banchmuskeln im Wochenbett.

Basis aufstellen. Dieselben möchte ich vorschlagen $\mathrm{zu}$ bezeichnen als

1) „Ueberanspannung der Muskeln“. Die Bauchmuskulatur ist durch Missverhältniss zwischen Raum und Inhalt oder durch zu starke Bauchpresse bei der Geburt in ihrer Elasticität stark herabgesetzt, aber ohne wesentliche Gewebsveränderung.

2) „Einfache Bauchschlaffheit", vielleicht auch ,seitliche“ zu bezeichnen: Verminderter Muskeltonus, mässige druckatrophische Zustände der transversalen Muskeln mit keiner oder nur geringer Betheiligung der Längsmuskeln.

3) „Hochgradige Bauchschlaffheit" oder ,allgemeine Relaxation": Atrophische Zustände sämmtlicher die Bauchwand bildenden muskulären und sehnigen Gewebe.

Das Krankheitsbild der Ueberanspannung charakterisirt sich am häufigsten, wenn überhaupt Symptome zur Klage kommen, durch Ermüdungsschmerzen in der Bauchmuskulatur, von den Frauen theils an die Grenze zwischen geraden und schrägen Muskeln hinten (Weiche), oder an dieselbe Stelle vorn verlegt, mitunter auch in die Recti selbst versetzt. Die Schmerzen lassen sich durch die Palpation als Muskelschmerzen von anderen unterscheiden. Ganz vorwiegend oft werden gar keine Klagen gefuhrt und nur die auch nach 12-24 Stunden post partum noch persistirende Diastase lenkt die Aufmerksamkeit des Arztes auf die Abweichung vom Normalen. Die Diastase ist. selten breit, aber dafür tief. Sie überschreitet selten $3-4 \mathrm{~cm}$ (Zweifingerbreite), aber man kann bei der vor der Geburt stark angespannten und nachher erschlafften Aponeurose oft drei und vier Finger senkrecht aufeinander gelegt in die Grube eindrücken. In diese Grube wölben sich die mitunter recht straff angespannten Recti mit dicken, wulstigen Rändern hinein. Die parietale Muskulatur ist intact erhalten, bei Lageveränderungen der Frau tritt eine typische Abhebung der Recti von derselben nicht ein. Dieser Befund ist neben der Norm der häufigste bei Erstgebärenden, bei Mehrgebärenden ist er fast noch häufiger, als der normale. Bei ruhiger Lage der Frau, genügender Defäcation und regulärer Genitalinvolution geht dieser Zustand im Laufe von sechs bis acht Tagen in den normalen über. Allein er birgt doch schon Gefahren, welche Beachtung verdienen. Bleibt der Uterus lange hochstehen, treten starke Anfüllungen ron Blase oder Darm, besonders Meteorismus, ein, so wird die Rückbildung gehindert, die Diastase be- 
steht länger, die schlaffe Linea alba wird von den Därmen oder dem Netze hervorgedrängt, und in Einzelfällen, wie wir sie oben schilderten, kann es sogar zu scheinbar bedrohlichen acuten Symptomencomplexen kommen. Weit häufiger wird die bestehen bleibende Diastase nicht beachtet, die Frauen stehen auf, verrichten Arbeit, gebrauchen die Bauchpresse stärker, der Bauchdruck wird wieder gegen die noch nicht geschlossene vordere und die nicht genügend gekräftigte seitliche Bauchwand hin verlegt und dadurch werden schnell Uebergänge zu den bei der einfachen Bauchschlaffheit zu schildernden Zuständen und Krankheitserscheinungen geschaffen.

Deshalb ist gerade dieses Verhalten der muskulären Ueberanspannung dasjenige, bei welchem am meisten prophylaktische Therapie angezeigt ist und Erfolg aufweist, zugleich dasjenige, bei dem die Rectaldiastase das wichtigste und unser Handeln bestimmende Moment abgiebt.

Bei der einfachen oder seitlichen Bauchschlaff heit sind bereits in verschieden hohem Grade atrophische Zustände der Muskeln, und zwar ganz vorwiegend der seitlichen, eingetreten. Man kann dies durch schwache Inductionsströme unter Einschaltung von Rheostaten ${ }^{1}$ ) bei Vergleichen an einer und derselben Person zu verschiedenen Zeiten oder Vergleichung gesunder und mit erschlafften Muskeln behafteter Wöchnerinnen direct nachweisen. Die Recti sind etwas verbreitert und abgeplattet, haben im Liegen nicht derbe, wulstige Ränder, sondern schlaffe Muskelbäuche, zeigen aber fast keine Veränderung ihrer Energie gegen den Inductionsstrom und contrahiren sich beim Erheben in normaler Weise. Zugleich heben sie sich dann in der schon früher erwähnten typischen Weise gegen die schlaffere transversale Muskulatur ab (s. S. 426). Die Linea alba ragt fast stets von vornherein etwas hervorgetrieben durch den Bauchinhalt über das Niveau der Muskeln hervor, die Diastase der Recti, welche sie hervorgebracht hat, ist eine breite und zugleich flache, zwar leicht eindrückbare, aber für gewöhnlich mehr hervorgewölbte. Die einfache Bauchschlaffheit betrifft Erstgebärende selten, und nur bei starkem Missverhältniss zwischen Bauchraum und Gebärmutter-

1) Ich habe eine Anzahl derartiger Untersuchungen an Wöchnerinnen und auch an Laparatomirten angestelit; leider gestattet die Privatpraxis nicht, dieselben in einem Maassstabe auszudehnen, welche sie einer Veröffentlichung werth erscheinen liessen. 
430 Prochownick, Die Diastase der Bauchmuskeln im Wochenbett.

inhalt, vorwiegend Mehrgebärende, auch bei nicht schneller Folge der Geburten. Mit besonderer Häufigkeit kommt sie bei Fettleibigen vor. ${ }^{1}$ ) Leider sind die subjectiven Erscheinungen und Beschwerden der Wöchnerinnen bei diesem Verhalten der Bauchwand in der grossen Mehrzahl aller Fälle, so lange die Frauen liegen, ganz auffallend geringe; selbst geringer als bei Ueberanspannung der Muskeln. Schmerzen fehlen, Anfüllung des Darmes mit Gasen, Obstipation, Blasenfüllung verlaufen dabei ohne Klagen von Seiten der Frauen. Nur selten leitet uns eine von der Wöchnerin geäusserte Neigung zu Flatulenz und Aufstossen, ein plötzlich eintretender Kolikschmerz nach Aufsitzen oder natürlichen Verrichtungen auf die richtige Fährte; am häufigsten noch springt die Unfähigkeit, die geschwächte Bauchpresse im Liegen in Thätigkeit zu setzen, ins Auge, indem Entleerung von Blase und Mastdarm in dieser Stellung stark behindert oder unmöglich ist, in anderen Stellungen aber, wenn der noch intacte Rectus die schwache Seitenmuskulatur zu beeinflussen oder zu ersetzen vermag, sofort erfolgt. Dementsprechend wird auf diesen $\mathrm{Zu}$ stand am wenigsten bei liegender Frau geachtet und der wichtigste Zeitpunkt, späteren Uebeln vorzubeugen, leicht verabsäumt. Ganz anders in verschiedenartiger Schnelligkeit, je nach Constitution und socialer Stellung der Frau, gestaltet sich das Befinden nach dem Aufstehen. Zuerst beschweren sich die Wöchnerinnen - denn so darf man sie doch noch bezeichnen - uiber Unbehaglichkeit im Magen, Vollsein nach Mahlzeiten, Schwere und Druck in der unteren Partie der Vorderbauchwand. Eine besonders wichtige Rolle spielen alsdann ,die Blähungen", denen diese und andere, selbst schwerere, Symptome zugeschrieben werden. Allmälig vermehrt sich der erst geringe Meteorismus; bei liegender Frau in den Symptomen fehlend und dem Auge kaum ersichtlich, stellt er sich sofort ein beim Herumgehen und zwischen den klaffenden Recti kann man an der aufrecht stehenden Frau mitunter Hervorstülpungen, ja selbst peristaltische Bewegungen von Darmpartien leicht erblicken. Das Druckgefühl nach unten vorn und die Empfindung des wegfallenden Leibes fast typisch, wenn nicht Genitalcomplicationen vorliegen, nach vorn über die Schamfuge hinweg, nimmt Schritt für Schritt zu, ver-

1) Es ist nicht unwahrscheinlich, dass rejchliche Fettbildung am Leibe auch für sich ohne vis a tergo Muskelschwund nach sich zieht; wenigstens ist mir bei einer Serie von Castrationen und Salpingotomien an Fettleibigen jedesmal die besondere Dünnheit der Muskeln, auch der Recti, auftällig gewesen. 
schieden schnell und verschieden hochgradig kommt es zum sogenannten Bauchbruche. Ueber diesen glaube ich in jedem Punkte auf die kurze, aber präcise Schilderung von Benno Schmidt im Pitha-Billroth'schen Lehrbuche ${ }^{1}$ ) verweisen zu dürfen, vielleicht mit dem Zusatze, dass bei Wöchnerinnen (und Laparatomirten) Bauchbrüche entschieden ebenso häufig unterhalb als oberhalb des Nabels vorkommen. Lange Dauer dieser Zustände oder erneute Schwangerschaft zieht ausnahmslos die ,hochgradige Bauchschlaffheit" oder ,allgemeine Relaxation" der Bauchdecken nach sich. Diese für die Leidenden und Aerzte gleich grosse Plage bedarf kaum einer Schilderung. Bei der Erschlaffung sämmtlicher die Bauchwand zusammensetzenden Gewebe treten die für die seitliche Bauchschlaffheit nach dem Aufstehen sich zeigenden Symptome schon gleich nach der Entbindung, oft genug auch bereits in der Schwangerschaft auf, nehmen auch bei Ruhelage im Puerperium eher zu als ab und erreichen ihre Höhe in den ersten Tagen nach dem Aufstehen. Die Stärke der Klagen ist allerdings subjectiv recht verschieden, manche Frauen ertragen hohe Grade von Schlaffheit mit wenig Schmerzäusserungen, ebenso oft aber wird dieses Leiden, noch dazu bei unserer therapentischen Ohnmacht, zur wahren Crux medicorum. Die Relaxation der Bauchdecken ist ausserdem in der Mehrzahl der Fälle mit dem gleichen Verhalten der Bindesubstanzen im Inneren des Abdomen vergesellschaftet und demgemäss recht oft mit Erschlaffungszuständen der Geschlechtsorgane verbunden. Es entstehen alsdann Krankheitsbilder, bei welchen es recht schwierig ist, die ursächlichen Bedingungen der geäusserten Klagen zu trennen und $z u$ erkennen. Eine ganze Serie auf die Genitalorgane bezogener „Senkungsbeschwerden“" weicht weder Pessarien noch Kolporraphien, sondern steht in Zusammenhang mit Erschlaffung der Bauchdecken und bessert sich mit fortgesetzt geduldiger Behandlung derselben, und umgekehrt gerathen wir in Versuchung, manche Klage, z. B. das Gefühl von Zug am Nabel, cardialgische Erscheinungen, Schmerzen vor und nach der Defäcation auf die Bauchschlaffheit ( - ganz gleich welchen Grades -) zu beziehen, und sie erweist sich doch bei exacter Untersuchung und mitunter erst ex therapia als rein genitalen Ursprunges. Hegar hat sich ${ }^{2}$ ) das Verdienst erworben, nicht nur auf diesen innigen Connex hinzuwei-

1) III. Bd. 2. Abth.

2) In dem Eingangs erwähnten Aufsatze. 
432 Prochownick, Die Diastase der Bauchmuskeln im Wochenbett.

sen, sondern auch einen Erklärungsversuch zu machen, indem er die mechanische Zerrung der in das Lendenmark einlaufenden Nerven bei Hängebauch und Bauchbruch das Centrum der Geschlechtsfunctionen beeinflussen lässt. Allein es wird noch der sorgfältigen Aufzeichnung vieler einzelner Thatsachen bedürfen, ehe wir auf diesem schwierigen Gebiete pathologisch aus den Vermuthungen und therapeutisch aus den leider nur zu oft erfolglosen Versuchen herauskommen. Gestehen wir wenigstens das Vorhandensein eines engen Zusammenhanges zwischen Bauchschlaffheit und gynäkologischen Krankheiten zu, so liegt darin eine Aufforderung mehr, der Heilung der Bauchschlaffheit unsere Aufmerksamkeit zu widmen.

Denn die Therapie ist bisher auf diesem Gebiete unsere schwächste Seite.

Vergegenwärtigen wir uns nun morphologisch mit den Anatomen, dass die gesammte Bauchwand eine Verlängerung des Thorax vorstellt, bei der das verlängerte Sternum von der Linea alba, die Rippen von den sehnigen Inscriptionen der Recti und die Intercostalmuskeln von den Obliqui repräsentirt werden, so können wir von vornherein in einem Punkte die Therapie generalisiren. Sie muss sich nämlich, wenn sie Erfolg bringen soll, auf diese sämmtlichen Partien, also auf die gesammte Bauchwand und nicht auf einzelne Theile erstrecken und sich möglichst fest auf den Halt des Ganzen, also auf die Wirbelsäule als Ausgangspunkt stützen. Vergegenwärtigen wir uns fernerhin die Krankheitserscheinungen, so kann als zweites Princip der Therapie aufgestellt werden, dass von Anfang an nicht die von den Wöchnerinnen angegebenen Symptome, sondern, ohne diese abzuwarten, lediglich die Befunde unser Handeln leiten sollen. Denn je mehr wir erkennen, dass in den vorbeugenden Maassregeln an uns selbst und an den uns anvertrauten Frauen der Fortschritt in der Behandlung von Geburt und Wochenbett beruht, um so mehr haben wir die Pflicht, bei der wachsenden Erkenntniss der wechselseitigen Beziehungen zwischen Bauchschlaffheit und Genitalerkrankung den Eintritt dieser Erschlaffungszustände nicht. abzuwarten, sondern ihn zu verhüten. Die Erfüllung dieser Pflicht wird uns glücklicherweise dort leicht gemacht, wo wir ärztlich das Puerperium controliren können, denn in der Mehrzahl der Fälle stehen eben palpable Befunde eher vor uns als Symptome, und es bedarf also nur der allgemeinen Einfuihrung des Gebrauches, täglich resp. bei jedem Besuche dem Verhalten der Bauch- 
wand bei der Wöchnerin eine Minute Aufmerksamkeit zuzuwenden, um gegebenen Falles vorbeugend therapeutisch einzugreifen.

Bei den leichteren Graden regelwidrigen Verhaltens der Bauchwand hatten wir die Diastase der Recti als das souveräne Kennzeichen aufgestellt. Die Rückkehr zur Norm findet vorwiegend von selbst statt, wenn nicht derselben entgegen gearbeitet wird. In manchen Gegenden Deutschlands nämlich wird mit Ausdauer der Leib der Frischentbundenen für Stunden und selbst Tage mit möglichst schweren Tüchern, Compressen, mit Sägespänen oder Sand gefüllten Säcken und dergleichen bedeckt. Diesem Missbrauche, der weder die Entfernung der Nachgeburt begünstigt, noch Blutungen hindert, noch der Involution günstig ist, den Bauchdecken aber, wie ich es mehrfach genau verfolgen konnte, zweifellosen Schaden bringt, muss zuvörderst ausgiebig durch Belehrung der Hebammen und Wärterinnen gesteuert werden. Selbst mit den gegen die Nachwehen mit Recht beliebten Priessnitz'schen Umschlägen muss man vorsichtig sein und die betreffenden Tücher immer möglichst wenig compact sein lassen. Wenn am sechsten bis siebenten Tage des Wochenbettes noch eine, alsdann als charakteristisch-pathologisches Symptom aufzufassende Rectaldiastase besteht, so müssen wir in Voraussicht der aus selbiger eventuell nach dem Aufstehen der Frau eintretenden Schäden vorbeugend therapeutisch vorgehen. Während wir von innen her durch reguläre Stuhlentleerung und Mittel gegen Gasanhäufung im Darme (Natron, Brausepulver, Wismuth, Pepsin, Mittelsalze u. s. w., je nach den individuellen Verhältnissen) eine Steigerung des inneren Bauchdruckes verhüten, suchen wir von aussen her durch einen mit mässigem Drucke vereinten Zug die seitlichen Muskeln und die Gesammtaponeurose zu entspannen, d. h. für unser Gefühl die Ränder der Recti mehr und mehr aneinander zu bringen. Für alle einfacheren Fälle genügen zu diesem Zwecke Leibbinden. Ueber die Art und Form dieser Binden liesse sich viel schreiben, jedoch wird nach meiner Ueberzeugung in diesem Punkte kaum je ein theoretisches Raisonnement zum Ziele führen, sondern immer der praktische Erfolg bei dem einzelnen Arzte für diese oder jene Bindenform entscheidend bleiben. Auch in England fand ich eine ganz beträchtliche Verschiedenheit in den theils von Aerzten, theils von Laien construirten Wochenbettsleibbinden vor. Am einfachsten und den Zwecken leichterer Fälle von Diastase völlig entsprechend erschien mir die Bindenanwendung in einigen grösseren 
434 Prochownick, Die Diastase der Bauchmuskeln im Wochenbett.

Gebärhäusern Londons. Beispielsweise sei gestattet, aus einem kleinen Taschenbuche, welches die DDr. John Williams und F. H. Champneys für die an ihrer Anstalt ') beschäftigten und von derselben in die Privatpflege entsandten Wärterinnen verfasst haben, den Abschnitt ,Anlegen der Binde“" in freier Uebersetzung wiederzugeben. ${ }^{2}$ )

„Die Binde soll aus einem damastartig gewebten Handtuchstoff bestehen, $92 \mathrm{~cm}$ breit und $125 \mathrm{~cm}$ lang sein, und wird in der ganzen Länge doppelt gelegt. ${ }^{3}$ ) Ihr unteres Ende reiche bis $10 \mathrm{~cm}$ unterhalb des Trochanter major und das freie Ende kommt hoch oben rechts zu liegen. Ausgehend von der linken Weiche lege man die Binde zunächst über den Leib, dann hinten um den Rücken herum und nochmals über den Leib hinweg. Nachdem sie nun fest angezogen und alle Falten ausgestrichen sind, befestigt man sie sorgfältig mit vier starken Nadeln. Man schütze die Haut der Wöchnerin mit der flach untergeschobenen linken Hand und steche die Nadeln folgendermaassen von unten her beginnend ein: die erste dicht über dem unteren Zipfel der Binde, $71 / 2 \mathrm{~cm}$ unter dem grossen Rollhügel, die zweite $71 / 2 \mathrm{~cm}$ unter dem höchsten Punkte der Crista ilei. Diese beiden Nadeln fassen die Binde fest zusammen, um sie in ihrer Lage festzuhalten und Hinaufgleiten zu verhïten. Die dritte Nadel legt man noch etwas fester anziehend in gleicher Höhe mit dem Gebärmuttergrunde an, und die vierte macht nahe dem oberen Ende der Binde etwas weniger fest fassend den Schluss. Gerade Nadeln von $10 \mathrm{~cm}$ Länge sind die besten."

Wie bereits Eingangs erwähnt, springen die Vortheile der Bindenbehandlung des Leibes im Wochenbette jedem vorurtheilsfreien Beobachter bei einer Vergleichung englischer und deutscher Frauenfiguren, besonders der Mittelstände, in die Augen, und eine Controle eigenen Materiales führt gleichfalls schon nach ein paar Jahren zur Erkenntniss, dass sich bei gleicher Aufmerksamkeit gleich günstige Resultate erzielen lassen. Noch mehr drängt

1) General Lying-in Hospital, York Road. Lambeth. S. E. Das kleine, kurzgefasste, solid gebundene Büchelchen umfasst in knapper, klarer Form alles für die Wärterin Wissenswerthe und kann zur Nachahmung dringend empfohlen werden.

2) Die englischen Maasse sind in Centimeter umgerechnet. 1 inch $=2,54 \mathrm{~cm}$.

3). Dies ist so zu verstehen, dass die Breite halb umgelegt wird und so die Binde doppelt macht; die eigentliche Breite kommt auf ca. $46 \mathrm{~cm}$ aus. 
sich die Klarheit darüber auf, dass selbst bei der seitlichen Bauchschlaffheit weniger ausgesprochenen Grades eine sehr frühzeitige Binden-Prophylaxe ausserordentlich günstig wirkt und das Eintreten schwerer Erscheinungen recht oft verhütet, wie mir auch englische Collegen nach ihren zum Theil sehr reichen Erfahrungen versicherten. Und dies führt uns zu dem Cardinalpunkte der Vorbeugungsbestrebungen: Sollen wir, wie es jenseits des Kanales geschieht, eine Bindenanlegung gleich nach der Entbindung allgemein einführen und sie durch Lehre und Uebung bei Hebammen und Wärterinnen fest einbürgern, oder sollen wir dieselbe nur bei objectiven oder eventuell subjectiven Symptomen an den Wöchnerinnen von ärztlicher Seite aus in Wirkung treten lassen?

Bei dem ungemein häufigen. Vorkommen der muskulären Ueberanspannung und der leichten Grade seitlicher Bauchschlaffheit, bei der Schwierigkeit, beide exact diagnostisch zu trennen, bei dem Bewusstsein, dass die grosse Mehrzahl dér Fráuen im Wochenbette und im Anschlusse an dasselbe bei uns niemals zu ärztlicher Beobachtung gelangt, brauchte man keinen Augenblick zu zweifeln, die vorbeugende Bindenanlegung nach der Entbindung allgemein in die Lehre und damit in den Gebrauch einzuführen. Dieser letztere würde sich auch bei der in den letzten Jahren verbesserten Hinneigung des Volkes zu einfachen hygienischen Maassregeln vermuthlich schnell einbürgern. Grundbedingung wäre, dass durch eine angelegte Binde in keinem Falle geschadet werden könnte. Nach den mir übereinstimmend mitgetheilten Erfahrungen englischer (und auch nordamerikanischer) Aerzte und den eigenen im Laufe der letzten Jahre gesammelten Beobachtungen darf meines Erachtens diese Grundbedingung bejaht werden. ${ }^{1}$ ). Es kann bei oben am Leibe zu straff angezogener Binde mitunter eine Belästigung der Wöchnerin (Oppression und Dyspnoe ähnlich wie bei Laparatomirten unter typischen Listerverbänden) vorkommen; dieselbe verschwindet stets mit Lockerung des betreffenden Bindenabschnittes; andere Nachtheile habe ich weder gesehen, noch wurden sie mir angegeben. Auch thut es der Bindentherapie keine Einbusse, dass sie nur in allen leichteren Fällen zum Ziele führt, denn eben diese sind die häufigsten und gestalten sich erst durch Mangel an Aufmerksamkeit resp. Prophylaxis zu schwereren.

1) Ich habe seit einigen Jahren erst versuchend und prüfend, dann allgemein die Bindenprophylaxis geübt.. 
436 Prochownick, Die Diastase der Bauchmuskeln im Wochenbett.

Diesen schwereren Fällen, also zunächst allen von scharf ausgeprägter seitlicher Schlaftheit gegenüber befinden wir uns in wesentlich schwierigerer Lage.

Beobachten wir in der zweiten Woche des Wochenbettes am achten bis zehnten Tage, also kurz vor dem Aufstehen mit oder ohne vorangegangene Bindenanlegung Erscheinungen, wie sie auf S. 429 n. 430 geschildert wurden, so genügt die einfache Binde zur unterstützenden Herstellung normaler Verhältnisse nicht. Wir müssen alsdann zu energischerem Drucke und Zuge übergehen, indem wir mit längeren Tüchern mehrfache Bindentouren um das Abdomen herumführen oder die Handtücher durch Flanellbinden oder Gazebinden mit Wattenunterlagen ersetzen. Bei diesen Verbänden sind Schenkeltouren zum Halte des Verbandes unerlässlich, zugleich aber für viele Frauen lästig. Kommt man mit denselben aus, was die palpatorischen Befunde bei dem täglich oder zweitägig vorzunehmenden Bindenwechsel ergeben, so müssen sie nach dem Aufstehen noch so lange, als es im einzelnen Falle nöthig erscheint, oft mehrere Wochen lang fortgesetzt werden. Sehr unterstützend kann bei den Bindenverbänden ein zweckmässig gleich nach dem Aufstehen angelegtes Schnürleib ${ }^{1}$ ) wirken; am besten eignen sich dazu die sogenannten Schwangerschaftscorsets, welche vorn und hinten, mitunter auch noch an seitlich eingelegten Keilstiicken schnürbar sind. Oft genug jedoch erweist sich der Bindenpanzer als ungenügend oder unbequem. Von Alters her ist dann Heftpflaster zum Verbande empfohlen worden. ${ }^{2}$ ) Aber gerade mit diesem wurde gefehlt, indem man, die Rectaldiastase als das Wichtigste ansehend, es einfach über die vordere Bauchwand in verschiedener seitlicher Ausdehnung hinwegspannte. In den seltensten und stets dann leichteren Fällen weist dieses Verfahren Erfolg auf, schon bei einigermaassen stärkerer Seitenschlaffheit kann man sich stets nach wenigen Stunden des Liegens von der völligen Nutzlosigkeit so angebrachter Heftpflasterstreifen überzeugen. ${ }^{3}$ ) Ganz anders gestaltet sich der Heft-

1) Dass die Anlegung eines Schnürrumpfes mit scharfgebogenen Stahlstäben und auf die Taille concentrirter Schnürung nicht zweckmässìg ist, liegt nahe, jedenfalls muss die mittlere Schnürung dann nur eine sehr geringe von Zeit zu Zeit zunehmende sein.

2) Siehe z. B. Jörg's Krankheiten des Weibes, S. 583. Leipzig 1821.

3) Das Gleiche gilt von der Heftpflasterverwendung bei Laparatomien; besonders wenn es nach Wegnahme der Fäden Nutzen bringen soll, muss es circulär angelegt werden; bei liegenden Fäden kommt man vielfach mit der Anlegung über die Vorderbauchwand aus. 
pflasterverband, wenn er, auf die Wirbelsäule gestütat, die Bauchwand von beiden Seiten her gleichmässig umfasst und vorn noch in genügendem Maasse gekreuzt ist. Derartige Verbände sind, da sich die einzelnen Streifen genau wie Bindentouren ohne Haut dazwischen zu fassen oder frei zu lassen decken müssen, durchaus nicht leicht anzulegen. Die Streifen sollen nicht schmal, sondern 4 bis $5 \mathrm{~cm}$ breit sein, die Länge muss man für jede Tour beliebig dem Bedürfniss anpassen können, es muss Tour um Tour von unten nach oben bis zu oder ein wenig über Nabelhöhe angelegt werden. Höher hinauf treten leicht Druckerscheinungen von Seiten des Magens oder Querdarmes ein; auch genügt diese Höhe beinahe stets. Man kann über die vorn sich kreuzenden Enden zur Verhütung des Aufrollens noch einige schmälere Verstärkungsrunden anlegen, welche vorn sich stïtzend nach hinten auslaufen oder über der Wirbelsäule gekreuzt werden. Es ist ebenso angebracht als unschädlich, gesunde Wöchnerinnen zum Anlegen dieser Verbände zwischen dem sechsten und neunten Tage für einige Minuten (mit Unterstützung) aufstehen zu lassen. Durch die vorzüglichen Verbesserungen der Heftpflaster in den letzten Jahren (z. B. von Unna-Beiersdorf in Hamburg und Seabury \& Johnson in London) ist die ganze Technik sehr erleichtert, besonders durch das ohne jede Nachhülfe sofort eintretende Haften dieser neueren Pflasterarten auf der Haut, und die in allen Längen und Breiten fertig vorhandenen Stuicke resp. Binden. Leider sind die Verbesserungen dieser Pflaster noch nicht soweit fortgeschritten, um nicht bei einzelnen Kranken Juckreiz, bei manchen sogar geringe Eczeme zu erzeugen; auch ist die Wiederentfernung mitunter schwierig und etwas schmerzhaft. ') Allein diese kleinen Nachtheile stehen in keinem Verhältnisse zu der prompten Wirkung eines sorgsam angelegten und nach Bedürfniss ein-, höchstens zweimal wiederholten Heftpflasterverbandes. Nach einer vorübergehenden Unbehaglichkeit von ein paar Stunden fühlen die Frauen selbst den guten Halt, welchen der Verband gewährt, und sind meistens in Kürze selbst von dem

1) Z. B. klebt das Pflaster von Seabury \& Johnson stets vorzüglich, ist aber schwer zu entfernen und macht kleine Eczeme; das Unna-Beiersdorf' sche ist reizlos und leicht zu entfernen, lässt aber mit der Klebkraft beim Anlegen, wenn es nicht ganz frisch ist, oft im Stich:. Die Entfernung geschieht am leichtesten unter Nachhülfe von in 0l. terebinth. getauchten Schwämmchen, mit denen man vor der Loslösung von der Haut das Pflaster befeuchtet. 
438 Prochownick, Die Diastase der Bauchmuskeln im Wochenbett.

ihnen damit gewährten Vortheile durchdrungen. 1) Auch diese Heftpflasterverbände ergänzt noch weiterhin verbessernd das bald nach dem Aufstehen leicht und zweckmässig angelegte Schnürleib. Der besondere Vorzug derselben aber beruht darin, dass sie auch bei schon eingetretenen Folgeerscheinungen der seitlichen Bauchschlaffheit (vergl. S. 430) und selbst bei mässigen Bauchbrüchen noch weitaus besšere Dienste leisten, als Leibbinden und Bindenverbände; freilich lässt sich alsdann die Wirkung, namentlich bei spätem Anlegen, nicht so vollständig erreichen, als bei der prophylaktischen Application. In einigen Fällen hochgradiger seitlicher Schlaffheit bei weiter Diastase und auch schon nicht mehr ganz intacten Recti vermochte ich durch drei bis fünf wiederholte Heftpflasterverbände noch so weit Besserung zu erzielen, dass der nach dem ersten (2 Mal) resp. zweiten (1 Mal) Wochenbette entstandene Bauchbruch im Anschluss an das darauffolgende Wochenbett, in dem der Verband angelegt ward, zum Verschwinden kam. Die Ränder der Recti wurden völlig genähert, die Ausbuchtung der weissen Linie verschwand und der gefürchtete „dicke Leib“" kam nicht wieder. Allerdings entwickelten die betreffenden Wöchnerinnen in Betreff der kleinen Beschwerden des mehrfach wiederholten Heftpflasterverbandes eine anerkennenswerthe Geduld.

Ausser diesen Verbänden resp. der Bindentherapie im Allgemeinen sind wir gegen die einmal eingetretenen Folgen der Bauchschlaffheit und zugleich gegen die (quoad therapiam gleichwerthige) allgemeine Relaxation der Bauchdecken recht ohnmächtig in unseren Heilbestrebungen. Ganz hervorragend gilt dies von den Frauen der arbeitenden Klasse, und selbst die schwachen Hülfsmittel passender Leibbinden müssen sich diese Frauen oft genug versagen. Unter Umständen bietet eine lange fortgesetzte Anwendung des Inductionsstromes recht gute Aussichten, und in Verbindung mit Milch- und Eisencuren vermag man, sorgfältig individualisirend, recht häufig noch mit Geduld Erfolge zu erreichen, welche von vornherein nicht $\mathrm{zu}$ erwarten standen. Besonders in den grösseren Städten, wo meistens jetzt leihweise Batterien zu haben sind, empfiehlt sich diese Behandlungsmethode.

1) Hier und da findet man Frauen, die Heftpflasterverbände, ja überhaupt alle Bindenverbände nicht vertragen; sie werden unruhig, kurzluftig, meteoristisch, bekommen Singultus u. s. w., und ruhen nicht, bis die Binde wieder abgenommen ist. Dasselbe geschieht besonders häufig, wenn sich Bauchschlaff heit mit Wanderniere verbindet, oder - wie mir schien - bei solchen Frauen, welche Deviationen der Wirbelsäule aufweisen. 
Man belehrt die Patienten über die Handhabung der Apparate, die entsprechend dem Falle geeigneten Ansatzpunkte der Elektroden und lässt ein bis zwei Mal am Tage längere Zeit hindurch (12 bis 15 Minuten) Ströme von solcher Stärke, dass sie eben kräftig-prickelndes Gefühl erzeugen, auf die Bauchmuskulatur wirken. Starke Ströme wirken leicht nachtheilig durch hervorgerufene Magen- oder Darmerscheinungen. Die Leibbinden, deren sich alle Frauen mit Bauchschlaftheit, wenn es ihre Verhältnisse irgend gestatten, bedienen sollten, selbst wenn sie nur zur Erleichterung der Beschwerden und zum Verhüten der Verschlimmerung getragen werden, sind aussergewöhnlich oft ein Klageobject der Frauen, weil sie schematisch oder fabrikmässig hergestellt sind. Die individuellen Verschiedenheiten sind aber so grosse, dass in beinahe jedem Einzelfalle irgend eine Besonderheit an den Binden anzubringen ist, und es muss sich zur Erreichung einer gut sitzenden und wirkenden Binde jedesmal das nachprüfende Auge des Arztes mit dem Geschicke des Bandagisten vereinigen. Die in den letzten Jahren hergestellten Binden (namentlich die von der W. Teufel'schen Fabrik in Stuttgart und anderwärts nach ähnlichen Grundsätzen gefertigten) bezeichnen im ganzen Schnitt und den Verstärkungsriemen, sowie in den auf Zusammenfassen der ganzen Bauchwandung gerichteten Schlussmechanismen wesentliche Fortschritte ${ }^{1}$ ) und sind prophylaktisch (besonders auch für Laparatomirte) recht brauchbar; allein bei den Frauen mit allgemeiner Relaxation bedürfen sie jedesmal noch der Anpassung an das Individuum. Meist ist ein Festhalten nach unten in irgend einer Weise benöthigt. Die sogenannten Schenkelriemen sind dann ein recht misslicher Nothbehelf. Weit günstiger wirken Schenkelstücken. Dieselben werden, das obere Drittel des Oberschenkels umfassend, aus Flanell oder Lasting hergestellt, sind zum Schnüren eingerichtet und werden durch Anknöpfen mit der Leibbinde in Verbindung gesetzt; das Ganze gewinnt dann ungefähr das Aussehen einer Badehose. Diese Schenkelstücke zeichnen sich besonders auch durch ihre Reinlichkeit, Geruchlosigkeit und Billigkeit aus, und die Patienten machen sich nach dem ersten vom Bandagisten hergestellten Modell eine ihnen beliebende Zahl derselben zum Wechseln. Bei Personen mit starker

1) Ich versuchte in England, mir eine Reihe derartiger Binden zu verschaffen resp. in Augenschein zu nehmen, muss aber gestehen, dass mir doch die Teufel'schen Modelle praktischer erschienen sind. 
440 Prochownick, Die Diastase der Bauchmuskeln im Wochenbett.

Beckenneigung, stark abfallenden Darmbeinschaufeln, grosser Magerkeit bei gleichzeitiger Bauchschlaffheit, so dass bei aufrechtem Stehen der Leib weit hinter einer die Hüftbeinstacheln und Schamfuge verbindenden Ebene zurücksteht, muss auch die Leibbinde selbst durch Verminderung der Höhe, Ausfütterung, Einlegung besonderer elastischer Keile u. s. w. genau den besonderen Verhältnissen angepasst werden.

Leider führt auch sorgfältige Individualisation in jedem Punkte der therapeutischen Versuche in recht vielen Fällen zu höchst unbefriedigenden Erfolgen, und man legt sich im Laufe der Behandlung von Bauchschlaffheit mehrfach die Frage vor, ob nicht auf chirurgischem Wege eine dauernde Herstellung $z \mathfrak{u}$ erzielen sei. Durch alle diesen Gegenstand betreffenden Veröffentlichungen zieht sich die Aufführung der von G. Simon und Hegar in dieser Richtung angestellten Versuche hindurch, allein dieselben haben entweder nicht zu ferneren Versuchen ermuthigend gewirkt, oder die eventuell gemachten sind nicht erfolgreich auf die Dauer gewesen, denn es ist bisher nichts weiter über dergleichen publicirt worden. Es muss auch, sowohl nach Analogie der sehr wenig befriedigenden Erfahrungen der Chirurgen mit Nabelbruchoperationen, als auch nach dem über die Genese der Bauchschlaffheit Gesagten zugegeben werden, dass die operative Annäherung der Recti, die Resection der erschlafften Aponeurose, für die Beseitigung eines durch Erschlaffung entstandenen Bauchbruches nur dann eine Aussicht auf dauernden Erfolg bieten kann, wenn alle Factoren zur Relaxation damit beseitigt wären, oder im Anschlusse an die Operation sicher beseitigt werden könnten. Dies aber ist durchaus nicht der Fall; und ob lediglich zur Beseitigung eines Symptomes operative Eingriffe, die leicht $\mathrm{zu}$ wirklicher Laparatomie unter den gegebenen Verhältnissen sich gestalten können, eventuell bei Wunsch von Erfolg sich gestalten müssten, am Platze sind, ist für jetzt noch offene Frage.

So führt denn gerade die geringe Leistungsfähigkeit unserer Heilmittel gegen die einmal bestehenden höheren Erschlaffungsgrade der Bauchwandungen zu der in jedem Einzelfalle wiederholten Ueberlegung zurïck, ob nicht rechtzeitige Aufmerksamkeit im Wochenbette und Anwendung vorbeugender Maassregeln mehr hätte bewirken können, als aller gegen die Folgezustände gerichteter therapeutischer Aufwand. Und je mehr man sich erst einmal von dem reellen Nutzen prophylaktischen Handelns überzeugt, 
Prochownick, Die Diastase der Bauchmuskeln im Wochenbett. 441

um so mehr wird man sich verpflichtet erachten, zu möglichst ausgedehnter Anwendung desselben anzuregen.

Die vorstehende Arbeit bezweckt lediglich diese Anregung. Da sie sich nur auf Beobachtungen und Studien aus der Privatpraxis stiitzt, so unterliegt sie den damit verbundenen Nachtheilen und Vorzügen. Erstere bestehen darin, dass die Ausnutzung des Materiales zur Schaffung klarer diagnostischer und differential-diagnostischer Momente und zum Aufbau scharfer klinischer Krankheitsbilder eine ganz ausserordentlich beschränkte ist; letztere beruhen auf der Möglichkeit, eine grössere Anzahl von Personen Jahre hindurch, oft bei drei und vier Wochenbetten und im Anschlusse an dieselben genau zu beobachten, wozu wiederum dem Kliniker sehr selten Gelegenheit wird. Vielleicht nehmen berufene Kräfte diejenigen Seiten dieser ohne Zweifel für die Pathologie und Therapie des Wochenbettes recht wichtigen Angelegenheit, welche allein reichliche klinische Beobachtung entcsheiden kann, erneut in Arbeit! 